\title{
Raport Badawczy
}

\section{Research Report}

\section{RB/6/2017}

\section{Supporting multicriteria} fuzzy decisions on the Forex market

\author{
P. Juszczuk, L. Kruś
}

Instytut Badań Systemowych Polska Akademia Nauk

Systems Research Institute Polish Academy of Sciences 


\section{POLSKA AKADEMIA NAUK}

\section{Instytut Badań Systemowych}

ul. Newelska 6

01-447 Warszawa

tel.: $\quad(+48)(22) 3810100$

fax: $\quad(+48)(22) 3810105$

Kierownik Zakładu zgłaszający pracę:

Dr hab. inż. Lech Kruś, prof. PAN 


\title{
Supporting Multicriteria Fuzzy Decisions on the Forex Market
}

\author{
P. Juszczuk* L. Kruś ${ }^{\dagger}$
}

\section{Abstract}

The paper deals with decisions made by a decision maker using technical analysis on the Forex market. For a number of currency pairs on the market a decision maker obtains buy or sell signals from transaction systems on the basis of technical analysis indicators. The signal is generated only when assumed conditions are satisfied for a given indicator. The information characterizing every market situation and presented to the decision maker is twofold. He obtains a respective signal or no.

In this paper a fuzzy, multicriteria approach is proposed to extend and valuate information for analysis of the market situation. The traditional approach with binary characterization of the market situations, considered as a crispy case, is replaced by a fuzzy approach. In the last case the strict conditions for which the crisp signal was generated are fuzzy. Efficiency of a given currency

* University of Economics; Faculty of Informatics and Communication; Chair of Knowledge Engineering, ul. 1 Maja 50, 40-287 Katowice, Poland.

† Systems Research Institute, Polish Academy of Sciences, Newelska 6, 01-447 Warsaw, Poland; 
pair is estimated in the range of $\langle 0,1\rangle$ values defined by a respective membership function for each technical indicator. The values calculated for different indicators are treated as criteria. The efficiency of a given currency pair can be analyzed jointly for several indicators. The currency pairs are compared in the multicriteria space in which domination relations are introduced describing preferences of the decision maker. An algorithm is proposed generating Pareto-optimal variants of currency pairs presented to the decision maker. The method proposed allows to extend number of analyzed currency pairs, without visibly increasing the computation time.

\section{Introduction}

Forex market is a global, decentralized currency market. It is referred as the most liquid market in the world, and its turnover even in the local currencies often reach trillions of dollars every day [12]. A currency pair is the basic instrument considered on the Forex market. Basically the currency pair may be considered as the ratio of one currency to another. Due to 4 overlapping sessions the Forex market has high accessibility even for retail clients. Popularity of the Forex market is related to easily accessible trading tools. Concepts like technical analysis (which includes trend indicators, oscillators, Fibonacci levels, Pivot points and more), along with fundamental analysis are nowadays crucial elements of rule-based trading systems, in which easily-understandable signals are used to open trading positions.

The rule-based trading system can be considered as a set of rules related with technical analysis indicators or candle formations which are transformed 
into the trading signals. Nowadays there is growing tendency, in which above concepts are included into various complex systems based on neural networks [18], [10], evolutionary prediction [16], evidence theory [11] and more. While the fuzzy sets are also included in these methods [8], there is a significant number of papers dealing with multi-agent systems [1]. Concepts of the AI systems [19] along with the evolutionary computation [4] are intensively extended. Articles concerned on the the fundamental analysis included into the trading systems are less popular than the systems based on the technical analysis [13].

Still growing number of instruments available for the decision maker, where, at the same time there is no agreement about efficiency of the technical analysis [2] (thus the same can be stated for the rule-based trading systems) creates a visible gap. Number of papers dealing with optimization of the technical indicators is sustainable high, while the papers like [2] indicate, that these concepts may be effective only in the very specific situations, or are not effective at all. It is not isolated work, and even in the case of the High Frequency Trading systems there is an observable tendency, that efficiency of automated trading systems decreases over time [15]. We propose to cover this gap with the use of a specially constructed system allowing to support the decision maker in the trading process. By the decision maker we understand trader or any retail client with access to the Forex market. Moreover his/her goal is to achieve the highest possible profit acquired from orders opened on the basis of signals generated from the set of trading rules. However nowadays we can see tendency, where the number of potential instruments available to the decision maker on the Forex market exceeds over 100 instruments. 
While terms "decision maker" and "trader" in the presented paper could be used simultaneously, we prefer to stick with the decision analysis terms thus further we use term "decision maker".

The approach introduced in this article allows to initially estimate the set of currency pairs potentially interesting for the decision maker. It should be clearly stated, that our motivation is only to support the decision maker, and not to present automatic trading system, so the main advantage of the proposed solution is that it assures sovereignty of the decision maker (trader). The final decision related to opening the transaction for the given currency pair belongs to the decision maker. Thus estimated set variants could be identified as the preliminary suggestions presented to the decision maker.

We propose an extension of the crisp approach currently used, where the signal is generated only in very specific market situations. In the crisp approach the decision maker has a limited time to open the transaction when the signal was generated. Thus two-fold signal mechanism based on binary "signal / no signal" values seems to be inefficient and in the case of large number of instruments often leads to the situation in which there is no single currency pair being potentially interesting to the decision maker.

In the fuzzy approach proposed here there is possibility to open a transaction in some predefined time interval related with the willingness to take the risk by the decision maker. Such approach guarantees, that the fuzzy approach is the generalization of the crisp approach, and the signals generated in the crisp approach are also included.

The outline of this work is as follows. After the introduction in Section 
2 the concept of the crisp approach commonly used on the Forex market is briefly described. Next the fuzzy approach along with definitions of the membership functions are proposed. Section 3 includes a description of the proposed dominance-based algorithm generating non-dominated variants for the decision makers. Section 4 presents preliminary experiments conducted on real-world data. Finally we conclude and derive directions of future works.

\section{Crisp and fuzzy systems}

In the classical crisp approach the rule-based trading system includes a predefined set of transaction rules related with the technical indicators. Every indicator can be described by the set of rules, which can be transformed into the binary activation function. A signal is generated only in the case, when the function value is equal to 1 . In the fully automated-trading system positive function value is equal to opening the transaction, while in the crisp decision support system information about the signal is derived in the system and presented to the decision maker.

We propose a fuzzy concept, in which information about a market situation is transformed into a value of the membership function for each considered indicator. Such value is calculated for every considered currency pair. So each currency pair in a given market situation in a time $t$ is represented in the analysis as a variant with the vector of criteria related to particular indicators. To estimate the efficiency of such approach we compare it with the classical crisp approach, where criteria for all indicators are built on the basis of the binary activation function. To describe accurately the proposed 
concept, we consider buy signals, however the same concept can be used in the case of short sells.

To be more specific we make use of two very popular technical indicators: the Relative Strength Index (RSI) and the Commodity Channel Index (CCI). The CCI indicator was originally proposed in the 1980' by Donald Lambert. The rules explaining the indicator are described in [6]. A description of the RSI indicator can be found in [17]. These indicators are based on the so called oversold and overbought levels. They are frequently used to predict potential price changes. An exemplary price chart with these indicators is given in Fig. 1. We can see the overbought and oversold levels in the upper and lower parts of the indicator windows. We used the default parameters for the indicators with the overbought levels equal to 100 (in the case of $\mathrm{CCI}$ ), and 70 (in the case of RSI). The oversold levels are equal to -100 and 30. In this particular example the trading rule may be considered as the situation, in which the indicator (CCI or RSI) crosses one of the levels defined above. In the case of crossing the oversold level upwards - the buy signal is generated. The sell signal is generated in the opposite case, when the overbought level is crossed downwards.

Crisp signal for the RSI and CCI indicators are given as follows:

$$
\begin{aligned}
& \operatorname{cond}_{R S I}=\text { true if }\left(R S I_{n}(t-1)<c_{1}\right) \wedge\left(R S I_{n}(t)>c_{2}\right), \\
& \text { cond }_{C C I}=\text { true if }\left(C C I_{n}(t-1)<c_{1}\right) \wedge\left(C C I_{n}(t)>c_{2}\right),
\end{aligned}
$$

where $R S I_{n}(t-1)$ is a $R S I$ value in the time $t-1$ for the period $n ; C C I_{n}(t-1)$ is a $C C I$ value in the time $t-1$ for the period $n ; c_{1}$ and $c_{2}$ are constant values related with their overbought and oversold levels. 


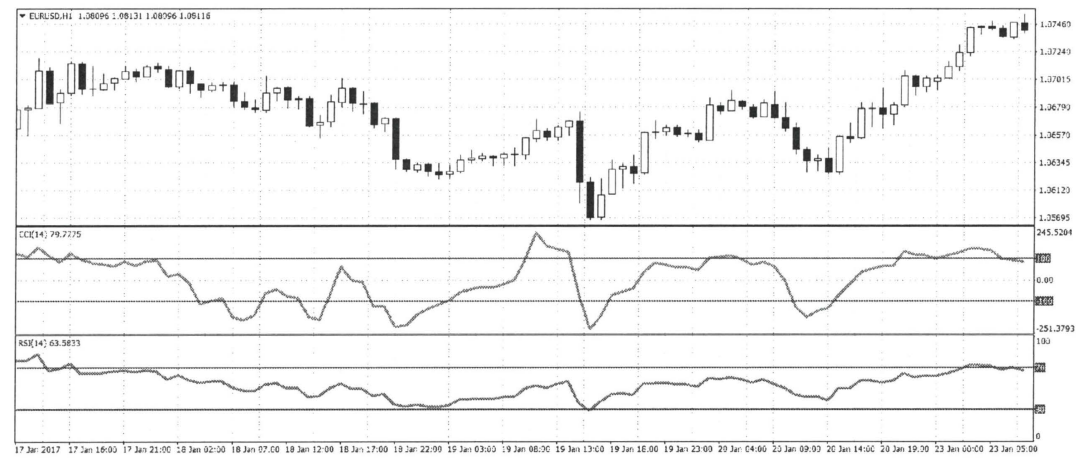

Figure 1 Example indicators and the price chart

We propose the fuzzy approach, where the original signal taken from the crisp approach is still included. However, the neighboring values of the indicator can be also included by calculation of the membership function:

$$
\mu_{R S I}(c)=\left\{\begin{array}{l}
\frac{R S I_{n}(t)}{30} \text { if }\left(R S I_{n}(t)<30\right), \\
1 \text { if }\left(\left(R S I_{n}(t-1)<30\right) \wedge\left(R S I_{n}(t)>30\right)\right) \\
\vee\left(R S I_{n}(t)=31\right), \\
\frac{0.9}{R S I_{n}(t)-30} \cdot \alpha \text { if }\left(R S I_{n}(t)>31\right) \\
\wedge\left(R S I_{n}(t)<50\right) \wedge\left(R S I_{n}(t-1) \leq 30\right), \\
0 \text { if }\left(R S I_{n}(t)>50\right) .
\end{array}\right.
$$

where $\alpha$ is a scalarizing factor in the range $\langle 0.5,1.1\rangle$ and $c$ denotes the currency pair for which the conditions on the right hand side of the equation are checked. The transaction system collects information from the market and calculates the values of the indicator at a given time $t$. Using the indicator the system checks the conditions and derives the membership function value. 
The membership function for the CCI indicator is calculated as follows:

$$
\mu_{C C I}(c)=\left\{\begin{array}{l}
0 \text { if }\left(C C I_{n}(t)<C C I_{\min }\right), \\
\frac{C C I_{n}(t)-C C I_{\min }}{-C C I_{\min }-100} \text { if }\left(C C I_{n}(t)>C C I_{\min }\right) \\
\wedge\left(C C I_{n}(t)<-100\right), \\
1 \text { if }\left(C C I_{n}(t-1)<-100\right) \wedge\left(C C I_{n}(t)>-100\right), \\
\frac{C C I_{n}(t)+50}{-50} \text { if }\left(C C I_{n}(t)>-100\right) \wedge\left(C C I_{n}(t)<-50\right) \\
\wedge\left(C C I_{n}(t-1)>-100\right), \\
0 \text { if }\left(C C I_{n}(t)>-50\right) .
\end{array}\right.
$$

where $C C I_{\min }$ is the minimal possible value of the indicator CCI and $C C I_{\max }$ is the maximal possible value of the indicator CCI. Basically in the case of the crisp approach a signal can be observed only in a specific time tick usually, when the indicator value is derived. In the most cases the rules in the crisp approach take into consideration two neighboring values of the indicator. When the relation between these two values is fulfilled (on the basis of the equation (1) or (2)) the signal is derived for the decision maker. In the case of the fuzzy approach the signal can be generated when the value of the membership function is higher than zero. Therefore the signal can be observed within a period longer than in the crisp approach and the decision maker has more time to made a decision.

\section{Dominance-based algorithm}

Let us consider the buy signals. The sell signals can be treated in the same way. Let $c$ be a currency pair valuated by a vector $y$ of two criteria $y=\left(y_{1}, y_{2}\right)$. Variants of the vectors are analyzed in the criteria space $\mathbb{R}^{2}$. The criteria refer to the RSI and CCI indicators with the values of membership functions: $y_{1}=\mu_{R S I}(c)$ and $y_{2}=\mu_{C C I}(c)$ for a given currency pair $c$. 
The transaction system generates some number of such variants in a given time window. By the time window we understand a time, that is needed to generate a new value on the price chart.

The decision maker, i.e. - trader, tries to find a variant with possibly maximal values of all the criteria; therefore the following relations between variants are considered in $\mathbb{R}^{2}$ space:

Definition 3.1. A variant $y$ is at least as preferred as a variant $z$ if each criterion of $y$ is not worse than the respective criterion of $z$.

$$
y \succeq z \Leftrightarrow\left(y_{1} \geq z_{1}\right) \wedge\left(y_{2} \geq z_{2}\right)
$$

Definition 3.2. A variant $y$ is more preferred (better) than a variant $z$ according to the logical formulae:

$$
y \succ z \Leftrightarrow(y \succeq z) \wedge \neg(z \succeq y)
$$

Definition 3.3. A variant $y$ is incomparable with a variant $z$ if

$$
\neg(y \succeq z) \wedge \neg(z \succeq y)
$$

The domination relation 6 defines a partial order in the space of criteria. We propose algorithm 1 deriving non-dominated variants which can be analyzed by the decision maker. The following notation is used in the algorithm.

- $Y$ denotes the set of all considered variants in a given time window.

- $u=(1,1)$ is assumed as the aspiration point of the decision maker. If there exists a variant equal to the aspiration point, it should be considered as the only rational choice for the decision maker. 
- $x$ is the reservation point assumed by the decision maker. All variants dominated by this point are removed from further analysis. It relates to the willingness of the decision maker to take a risk by extending the set of accepted variants in comparison to the crisp approach. To be more specific, greater risk leads to possibility of accepting potentially worse variants instead of delivering the empty set of variants to the decision maker.

- $N D$ denotes the set containing all non-dominated variants potentially interesting for the decision maker in a given time window.

- $Y_{-}$is the set of points removed from analysis in the algorithm, initially the points dominated by $x$ i.e. $Y_{-}=\left(x+\mathbb{R}_{-}^{2} \backslash\{0\}\right)$, where $\mathbb{R}_{-}^{2}$ is the negative cone. Further, it is the set of all points dominated by $x$ and the variants currently included to $N D$.

- $Y_{+}$denotes the set of points accepted for further analysis in the algorithm, $Y_{+}=Y \backslash Y_{-}$.

The algorithm, called the Dominance-based algorithm, enables generation of all non-dominated variants in the initial $Y_{+}$set accepted for analysis on the basis of the reservation point $x$ defined by the decision maker.

Algorithm steps can be divided into three separate phases. In the first phase the set $Y$ is derived by calculations of the criteria - membership functions for currency pairs in the assumed time window. At the same time, the sets $Y_{-}$and $Y_{+}$are derived on the basis of reservation point $x$ (lines 1-3). In the second phase (lines 4-5) variants equal to the aspiration point are looked for. If such a variant/variants exists, the algorithm is stopped and the resulting 


\section{Algorithm 1: Dominance-based algorithm}

\section{begin}

$1 \quad$ Fix the aspiration point $u$, create the set $Y$ and set $N D=\emptyset$

$2 \quad$ Decision maker sets the point $x$ defining the nonaccepted variants

3 Derive sets $Y_{-}$and $Y_{+}$

$4 \quad$ if there exists $y \in Y$ such, that $y=u$ then

for each variant $y$ in $Y_{+}$do

\section{if $y \in Y_{-}$then}

$\left\lfloor\right.$ Delete $y$ from further analysis, i.e. $Y_{+}=Y_{+} \backslash\{y\}$

\section{else if $y \notin Y_{-} \wedge N D=\emptyset$ then}

$\left\lfloor\right.$ Add $y$ to the set $N D$ and Update the set $Y_{-}$and $Y_{+}=Y_{+} \backslash\{y\}$. else

\section{for $z \in N D$ do}

\section{if $y \succ z$ then}

Delete $z$ from $N D$

\section{else if $z \succ y$ then}

L Mark $y$ as dominated, delete it from $Y_{+}$, and BREAK

if $y$ is non-dominated then

Add $y$ to $N D$

Update the set $Y_{-}=Y_{-} \cup\left(y+\mathbb{R}_{-}^{2} \backslash\{0\}\right)$

Delete $y$ from further analysis, i.e. $Y_{+}=Y_{+} \backslash\{y\}$

\section{if $Y_{+}=\emptyset$ then}

end the algorithm 
set $N D$ includes only these variants to be selected by the decision maker as his obvious rational choice. The third most complex phase of the algorithm includes lines $6-18$. Three situations can be observed: first - if a selected variant $y$ is included in the set $Y_{-}$then it is removed from the analysis; second - if the set $N D$ is empty, then the variant $y$ is added to the set $N D$. The third situation occurs, when the set $N D$ is not empty. In such a case the variant $y$ is compared with every variant in the set $N D$. The variants from the set $N D$ dominated by $y$ are removed from the set $N D$ and $y$ is added to the set $N D$. If the variant $y$ is dominated by any variant of the $N D$ - the variant $y$ is removed from analysis. After any of these three situations set $Y_{-}$is updated in such way, that the area which it covers is expanded by the negative cone moved to the point $y$.
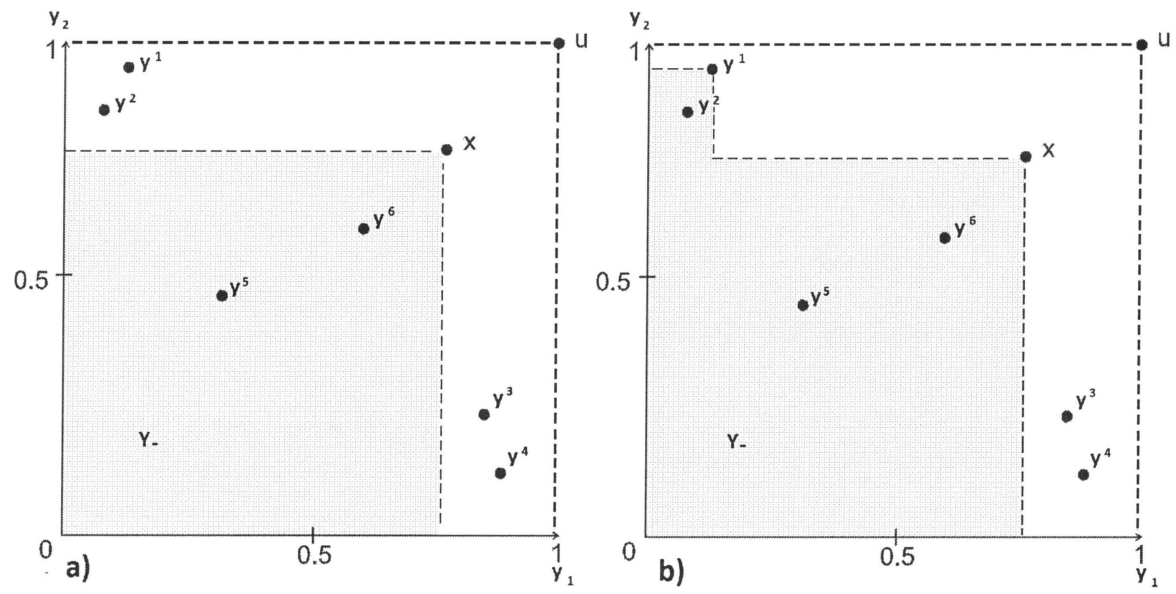

Figure 2 Illustrative example

An illustrative example is presented in Figure 2. In the part a) of the figure an initial simple situation is shown with a given reservation point $x$ and a 
set $Y$ consisting of 6 variants. The variants $y^{5}$ and $y^{6}$ are removed from further analysis because they are dominated by the reservation point $x$, as they belong to the initial $Y_{-}$set presented by the grey area. An analysis of four remaining variants $\left(y^{1}, y^{2}, y^{3}\right.$ and $\left.y^{4}\right)$ is illustrated in Fig. 2 b). At the beginning, the set $N D$ is empty, and the variant $y^{1}$ is not inside the grey area, thus it is added to the set $N D$. The set $Y_{-}$is expanded as follows:

$$
Y_{-}=Y_{-} \cup\left(y^{1}+\mathbb{R}_{-}^{n} \backslash\{0\}\right) .
$$

After updating the set $Y_{-}$, the variant $y^{2}$ is an element of the set $Y_{-}$, thus it is excluded from further analysis. Both variants $y^{3}$ and $y^{4}$ are incomparable with each other and incomparable with the variant $y^{1}\left(y^{1}\right.$ is already in the $N D$ set). In this particular scenario both the variants are added to the set $N D$. The grey area illustrating the set $Y_{-}$is expanded once again. There are no more variants left, thus the algorithm is finished. All non-dominated variants $\left\{y^{1}, y^{3}, y^{4}\right\}$ are given in the set $N D$ and can be presented to the decision maker.

\section{Preliminary experiments}

For the tests with real data we have selected overall 68 variants (currency pairs) from the January 2017. We tested three time windows: 5 minutes (the case of high frequency trading), 1 hour (the intraday trading), 1 day (the longterm trading). For every considered time window we have assumed three different positions of the reservation point in the criteria space: $x=(0.25,0.25)$, $x=(0.5,0.5)$ and $x=(0.75,0.75)$. For every considered combination of the 
above parameters (time window and reservation point position) we included 15 successive readings. The overall time of the experiments was equal to the length of the single time window multiplied by the number of readings. By the reading we understand a single situation on the price chart which is generated in the specific time window. To simplify: every new situation on the price chart corresponds to the new reading. The computation time for the single reading (including the generation of the non-dominated set $N D$ ) was approximately equal to 2 seconds.

First of all we derived variants which were not dominated by the reservation point. Numbers of such variants are presented in Table 1. They allow to estimate potential number of variants (included in the set $Y_{+}$) which must be analyzed in details. The table presents cardinality of the set $Y_{+}$for different time windows and different positions of the reservation point $x$. Lower values of the components of the point reservation point $x$ indicate a higher willingness of the decision maker to take the risk. At the same time the cardinality of the set $Y_{+}$is increased.

After the preliminary selection of the variants included in analysis, all the non-dominated variants were derived at the end of the given time window. The whole procedure was repeated for 15 successive readings. The results the cardinality of the set $N D$ for the time window 5 minutes are presented in Table 2. The number of non-dominated variants derived for different positions of the reservation point are decreasing when the position of the point is moved in the direction of the aspiration point $u$. In the case of the crisp approach the results obtained are presented in the last column of Table 2. It should be noted, that the rows containing the same numbers 
Table 1 Number of variants analyzed by the system with two indicators (RSI and $\mathrm{CCI}$ )

\begin{tabular}{|c|c|c|c|c|c|c|c|c|c|}
\hline & \multicolumn{3}{|c|}{ M5 } & \multicolumn{3}{c|}{ H1 } & \multicolumn{3}{c|}{ D1 } \\
\hline \hline $\mathrm{x}=$ & $\mathbf{0 . 2 5}$ & $\mathbf{0 . 5}$ & $\mathbf{0 . 7 5}$ & $\mathbf{0 . 2 5}$ & $\mathbf{0 . 5}$ & $\mathbf{0 . 7 5}$ & $\mathbf{0 . 2 5}$ & $\mathbf{0 . 5}$ & $\mathbf{0 . 7 5}$ \\
\hline \hline Reading 1 & 15 & 14 & 9 & 15 & 8 & 6 & 18 & 13 & 9 \\
\hline Reading 2 & 15 & 14 & 11 & 16 & 14 & 6 & 14 & 8 & 7 \\
\hline Reading 3 & 22 & 16 & 12 & 22 & 19 & 13 & 20 & 13 & 11 \\
\hline Reading 4 & 25 & 23 & 19 & 29 & 25 & 19 & 19 & 17 & 12 \\
\hline Reading 5 & 33 & 28 & 23 & 27 & 24 & 23 & 23 & 19 & 11 \\
\hline Reading 6 & 26 & 19 & 16 & 12 & 11 & 9 & 30 & 22 & 16 \\
\hline Reading 7 & 21 & 18 & 14 & 18 & 13 & 6 & 29 & 26 & 19 \\
\hline Reading 8 & 20 & 11 & 10 & 24 & 20 & 17 & 37 & 31 & 20 \\
\hline Reading 9 & 17 & 14 & 7 & 21 & 16 & 10 & 33 & 28 & 27 \\
\hline Reading 10 & 22 & 15 & 12 & 21 & 13 & 8 & 25 & 22 & 17 \\
\hline Reading 11 & 15 & 10 & 3 & 25 & 20 & 17 & 23 & 20 & 17 \\
\hline Reading 12 & 11 & 11 & 9 & 27 & 21 & 19 & 20 & 16 & 13 \\
\hline Reading 13 & 19 & 15 & 10 & 19 & 15 & 12 & 25 & 18 & 11 \\
\hline Reading 14 & 22 & 20 & 15 & 21 & 16 & 11 & 33 & 22 & 22 \\
\hline Reading 15 & 17 & 13 & 9 & 27 & 22 & 18 & 20 & 15 & 15 \\
\hline
\end{tabular}

for all column (Reading 2, 4 and 10) indicate the cases, when variants equal to the aspiration point were found. In these particular cases, such variants are presented to the decision maker as the only rational choices. Especially interesting are the cases, in which number of variants available for the decision makers is small. They can be observed in reading 11 and 15, where in the first case the crisp approach generated no variant at all, while the fuzzy approach generated two variants for all the considered values of $x$. In the second case, the crisp approach generated only one variant, while the number of variants derived on the basis of the fuzzy approach was two for $x=0.75$ and three for $x=0.5$ and $x=0.25$. In general the set of solutions generated in the case of the crisp method contains only the variants which were found in the corners 
of criteria space - where the membership function for one of the indicators takes his value equal to 1 . The fuzzy approach derives all non-dominated variants generated in the case of the crisp approach. It allows also to extend the set of acceptable variants by the non-dominated variants for which a deviation from the aspiration point $u$ is limited by the reservation point $x$. Namely the non-dominated variants belong to the set $\left(u+\mathbb{R}_{-}^{n}\right) \backslash\left(x+\mathbb{R}_{-}^{n} \backslash\{0\}\right)$.

Table 2 Number of non-dominated variants generated in the case of fuzzy approach in comparison to the number of variants generated in the crisp approach - 5 minutes time window

\begin{tabular}{|c|c|c|c|c|}
\hline & \multicolumn{4}{|c|}{ M5 } \\
\hline \hline $\mathrm{x}=$ & $\mathbf{0 . 2 5}$ & $\mathbf{0 . 5}$ & $\mathbf{0 . 7 5}$ & Crisp \\
\hline \hline Reading 1 & 7 & 7 & 5 & 2 \\
\hline Reading 2 & 1 & 1 & 1 & 1 \\
\hline Reading 3 & 12 & 9 & 9 & 5 \\
\hline Reading 4 & 3 & 3 & 3 & 3 \\
\hline Reading 5 & 18 & 16 & 14 & 9 \\
\hline Reading 6 & 9 & 8 & 7 & 3 \\
\hline Reading 7 & 8 & 8 & 8 & 6 \\
\hline Reading 8 & 8 & 7 & 5 & 3 \\
\hline Reading 9 & 5 & 5 & 3 & 2 \\
\hline Reading 10 & 1 & 1 & 1 & 1 \\
\hline Reading 11 & 2 & 2 & 2 & 0 \\
\hline Reading 12 & 7 & 7 & 7 & 4 \\
\hline Reading 13 & 10 & 8 & 7 & 5 \\
\hline Reading 14 & 10 & 9 & 8 & 5 \\
\hline Reading 15 & 3 & 3 & 2 & 1 \\
\hline
\end{tabular}

In Table 3 we present similar results of the cardinality of the $N D$ set for the time window equal to 1 hour. Once again the comparative results for the crisp approach were given in the last column. In the reading 2, 7 and 
14 once again number of variants derived in the case of the crisp approach was very small, while application of the fuzzy method increased number of non-dominated variants derived for the decision maker. A weak spot of the system can be observed in the case of relatively large number of variants generated by the crisp approach. This particular situation can be observed in the readings 5, 12 and 13, where the advantage of the fuzzy approach is not visible due to large number of variants derived for the decision maker on the basis of the crisp approach. In such situations an analysis based on a greater number of indicators should be made. In the case of large number of non-dominated variants generated by the fuzzy approach a respective ranking method should be applied.

Finally, the results for the longest time window considered on the Forex market as the long-term trading (1 day time window) which covered approximately three weeks from the January 2017 are presented in Table 4. Once again, the most useful information from the point of the view of the decision maker is derived for the example of reading 2, where the crisp approach allowed to obtain only one variant, while in the case of the fuzzy approach at least 3 variants were generated.

\section{Conclusions}

In this article we present an extension of the classical crisp concept used in the rule-based trading systems on the Forex market. The approach proposed delivers a opportunity to expand the set of variants possibly interesting for the decision maker. The fuzzy concept is presented as a generalization of the 
Table 3 Number of non-dominated variants generated in the case of fuzzy approach in comparison to the number of variants generated in the crisp approach - 1 hour time window

\begin{tabular}{|c|c|c|c|c|}
\hline & \multicolumn{4}{|c|}{ H1 } \\
\hline \hline $\mathrm{x}=$ & $\mathbf{0 . 2 5}$ & $\mathbf{0 . 5}$ & $\mathbf{0 . 7 5}$ & Crisp \\
\hline \hline Reading 1 & 6 & 5 & 5 & 3 \\
\hline Reading 2 & 3 & 3 & 2 & 1 \\
\hline Reading 3 & 6 & 6 & 5 & 3 \\
\hline Reading 4 & 2 & 2 & 2 & 2 \\
\hline Reading 5 & 20 & 20 & 19 & 17 \\
\hline Reading 6 & 8 & 7 & 6 & 4 \\
\hline Reading 7 & 5 & 5 & 4 & 2 \\
\hline Reading 8 & 14 & 10 & 10 & 7 \\
\hline Reading 9 & 9 & 7 & 6 & 4 \\
\hline Reading 10 & 5 & 5 & 5 & 2 \\
\hline Reading 11 & 9 & 8 & 7 & 4 \\
\hline Reading 12 & 16 & 14 & 12 & 10 \\
\hline Reading 13 & 15 & 13 & 11 & 10 \\
\hline Reading 14 & 7 & 7 & 5 & 3 \\
\hline Reading 15 & 1 & 1 & 1 & 1 \\
\hline
\end{tabular}

concepts commonly used in the rule-based trading systems. Along with the implementation of fuzzy membership functions, an algorithm deriving the set of non-dominated solutions is presented. The algorithm is specially useful in the case when the traditional crisp approach generates no signals at all, but the fuzzy approach gives some variants with the membership function close to 1 . The reservation point concept is related to the risk aversion of the decision maker.

The approach proposed assures full sovereignty of the decision maker. He decides how far he want to extend the set of variants analyzed by the system in comparison to the crisp approach. He obtains the generated non-dominated 
Table 4 Number of non-dominated variants generated in the case of fuzzy approach in comparison to the number of variants generated in the crisp approach - 1 day time window

\begin{tabular}{|c|c|c|c|c|}
\hline & \multicolumn{4}{|c|}{ D1 } \\
\hline \hline $\mathrm{x}=$ & $\mathbf{0 . 2 5}$ & $\mathbf{0 . 5}$ & $\mathbf{0 . 7 5}$ & Crisp \\
\hline \hline Reading 1 & 2 & 2 & 2 & 2 \\
\hline Reading 2 & 4 & 3 & 3 & 1 \\
\hline Reading 3 & 10 & 8 & 7 & 5 \\
\hline Reading 4 & 9 & 9 & 7 & 5 \\
\hline Reading 5 & 7 & 7 & 5 & 3 \\
\hline Reading 6 & 9 & 8 & 4 & 2 \\
\hline Reading 7 & 13 & 12 & 12 & 5 \\
\hline Reading 8 & 9 & 8 & 8 & 4 \\
\hline Reading 9 & 6 & 6 & 6 & 6 \\
\hline Reading 10 & 10 & 10 & 9 & 6 \\
\hline Reading 11 & 1 & 1 & 1 & 1 \\
\hline Reading 12 & 11 & 10 & 10 & 5 \\
\hline Reading 13 & 2 & 2 & 2 & 2 \\
\hline Reading 14 & 9 & 9 & 8 & 3 \\
\hline Reading 15 & 11 & 10 & 10 & 7 \\
\hline
\end{tabular}

variants. The decision maker decides which variant he will use to make a position.

The proposed approach is flexible. It can be used with different time windows and even with the different set of instruments. A position of the reservation point in the criteria space can be changed in every new reading. The approach presented for two indicators can be easily developed to handle with a greater number of them. A greater number of indicators included in the system should significantly reduce the set of non-dominated variants. Especially interesting may be indicators like the moving average [5], money flow index, ichimoku and others [14]. 
At the preliminary stage of experiments we assumed, that there are no complex dependencies between the considered two indicators. However in general for greater number of different indicators it is not strictly true. There are some indicators, which should be analyzed jointly taking into account some additional assumptions. Complex dependencies and complex transaction systems are planed to be introduced in the proposed method. Finally, deriving the large and difficult set of non-dominated variants naturally forces to present ranking of the generated variants which could greatly improve the process of final analysis made the decision maker. A respective ranking method is planned to be included in the system. Different ranking approaches are discussed including the ideas based on the concession line concept [7], the dominance-based rough set approach [3], the bipolar method [9].

As it was mentioned before, the presented approach delivers an initial simple version of the system, which can be expanded in multiple ways. Observing the increasing number of articles dealing with still developed more complex systems based on the technical analysis indicators, two distinct extensions seems to be especially interesting. Both are related with social phenomena of developed systems. The first extension assumes introduction of the fundamental analysis translated into easily understandable numeric values of the fundamental indicators. The second one is strictly related with the social trading considered as the mechanism for the collaborative trading on the market. Effectiveness of the social trading and systems based on the social networks (like twitter) is particularly difficult to estimate. On the other hand there are some possibilities to estimate activity of traders with the use of concepts like gamification. Therefore these concepts will be also discussed 
in the further works.

\section{References}

[1] R. P. Barbosa, O. Belo, Autonomous Forex Trading Agents, Advances in Data Mining. Medical Applications, E-Commerce, Marketing, and Theoretical Aspects, Lecture Notes in Computer Science, Vol 5077. Springer, 2008.

[2] P. Cheol-Ho, S. H. Irwin, What do we know about the profitability of technical analysis?, Journal of Economic Surveys, Vol. 21, Is. 4, Pages 786-826, 2007.

[3] S. Greco, B. Matarazzo, R. Słowińnski, Multicriteria classification by dominance-based rough set approach, Handbook of Data Mining and Knowledge Discovery, Oxford University Press, New York, 2002.

[4] A. Hirabayashi, C. Aranha, H. Iba, Optimization of the trading rule in foreign exchange using genetic algorithm, Proceedings of the 11th Annual conference on Genetic and evolutionary computation GECCO '09, Pages 1529-1536, 2009.

[5] C. C. Holt, Forecasting seasonals and trends by exponentially weighted moving averages, International Journal of Forecasting, Vol. 20, Is. 1, pp. 5 $-10,2004$.

[6] http://www.investopedia.com/articles/trading/05/041805.asp, access 10 IV 2017. 
[7] P. Juszczuk, I. Kaliszewski, D. Podkopaev, S. Hsu-Shih, Market Collective Wisdom Discovery for Portfolio Investments, International Journal of Information and Management Sciences, Vol. 27, Pages 87 ? 102, 2016.

[8] A. Kablan, Adaptive Neuro Fuzzy Inference Systems for High Frequency Financial Trading and Forecasting, Advanced Engineering Computing and Applications in Sciences, ADVCOMP '09, 2009.

[9] E. Konarzewska-Gubała, BIPOLAR: Multiple Criteria Decision Aid Using Bipolar Reference System, LAMSADE. "Cashier et Documents" 56, Paris, 1989.

[10] K. K. Lai, L. Yu,S. Wang, A Neural Network and Web-Based Decision Support System for Forex Forecasting and Trading, Data Mining and Knowledge Management, Lecture Notes in Computer Science, Vol 3327, Springer, 2005.

[11] Z. Liu, D. Xiao, An Automated Trading System with Multi-indicator Fusion Based on D-S Evidence Theory in Forex Market, Fuzzy Systems and Knowledge Discovery, FSKD ’09, 2009.

[12] G. McLeod, Forex Market Size: A Traders Advantage, dailyFx, January 24, https://www.dailyfx.com/forex/education/trading Access: 25 IV 2017, 2014.

[13] A. K. Nassirtoussi, S. Aghabozorgi, T. Y. Wah, D. C. Ling Ngo, Text mining of news-headlines for FOREX market prediction: A Multi-layer Dimension Reduction Algorithm with semantics and sentiment, Expert Systems with Applications, Vol. 42, Is. 1, Pages 306-324, 2015. 
[14] M. Patel, Trading with Ichimoku clouds: the essential guide to Ichimoku Kinko Hyo technical analysis, Wiley \& Sons, 2010.

[15] J. P. Serbera, P. Paumard, The fall of high-frequency trading:A survey of competition and profits, Research in International Business and Finance, Vo. 36, Pages 271-287, 2016.

[16] K. Slany, Towards the Automatic Evolutionary Prediction of the FOREX Market Behaviour, Adaptive and Intelligent Systems, ICAIS '09, 2009.

[17] W. Wilder, New Concepts in Technical Trading Systems, Trend Research, 1978.

[18] J. Yao, Ch. L. Tan, A case study on using neural networks to perform technical forecasting of forex, Neurocomputing, Vol. 34, Is. 1-4, Pages 79$98,2000$.

[19] L. Yu, K. K. Lai, S. Wang, Designing a hybrid AI system as a forex trading decision support tool, Tools with Artificial Intelligence, ICTAI '05, 2005. 


- 